\title{
Paper Complex Signal Mapping for Improving Spectral Efficiency of Li-Fi Systems
}

\author{
Ibrahim K. Sileh, Ayad A. Abdulkafi, Mohammed K. Hussein, and Saad M. Hardan \\ Electrical Engineering, Tikrit University, Salahaddin, Iraq
}

https://doi.org/10.26636/jtit.2019.131719

\begin{abstract}
Light fidelity (Li-Fi) systems based on the orthogonal frequency division multiplexing (OFDM) scheme have gained more attention in the communications circles, as a means to provide high spectral efficiency and ensure stricter data rate requirements for visible light communication (VLC) systems. However, common OFDM schemes employ Hermitian symmetry to obtain a real-valued signal which is necessary in intensity modulation and direct detection (IM/DD) optical systems, at the expense of doubling the required bandwidth. In this paper, a novel transmission scheme for an OFDM-based Li-Fi system is proposed to tackle the issue in question. A new approach to complex signal mapping (CSM), based on the pairing function method, has been developed for Li-Fi systems. It does not require Hermitian symmetry and, hence, saves about $50 \%$ of the required bandwidth. Unlike existing OFDM-based VLC approaches, the proposed scheme employs CSM to ensure a real and positive signal without Hermitian symmetry in order to fully utilize the bandwidth available to Li-Fi networks. Simulation results show that the proposed scheme significantly outperforms other systems in terms of spectral efficiency. The CSM-OFDM based Li-Fi system also achieves a good peak-to-average power ratio (PAPR) reduction with acceptable bit-error-rate (BER) performance, compared to conventional approaches.
\end{abstract}

Keywords-Li-Fi, OFDM, signal mapping, PAPR, BER.

\section{Introduction}

Light fidelity (Li-Fi) technology is an emerging sector on the optical communications arena. It relies on visible light communication (VLC) systems using light generated by light-emitting diodes (LEDs) to transmit data wirelessly. This technology may be useful in supporting and complementing the existing Wi-Fi systems, since its spectrum bandwidth is much broader. Higher data rates and capacities, accommodating more users, can be provided as well. Report [1] states that the $\mathrm{Li}-\mathrm{Fi}$ market is predicted to rise to reach the value of USD 75.5 billion by 2023, with an annual growth rate of 77\%. Along with that, ITU [2] and IEEE [3] have established first generation standards for VLC systems. Several vendors, such as Philips, PureLiFi, etc., have already produced first Li-Fi modems as well.
As LEDs have a signaling bandwidth of limited to several megahertz, orthogonal frequency division multiplexing (OFDM) may be considered a suitable candidate for LED-based Li-Fi systems due to its robustness to timevarying multipaths, its capacity to combat inter-symbol interferences (ISI), its high spectral efficiency and simple equalization [4]. Since the modulating signals of LED have to be real-valued and non-negative in intensity modulation and direct detection (IM/DD) systems, OFDM has to be modified accordingly. The most popular formats are DC-biased optical OFDM (DCO-OFDM) [5], and asymmetrically clipped OFDM (ACO-OFDM) [4]. In these forms, the input data vector of inverse fast Fourier transform (IFFT) has to be constrained to have Hermitian symmetry by adding the conjugate of the complex signal before the IFFT [4]. This ensures a real-valued time domain signal, but also reduces spectral efficiency and doubles the required bandwidth.

In the DCO-OFDM case, the non-negative signal is ensured after adding a DC-bias to the signal. However, due to the high peak-to-average power ratio (PAPR) of OFDM, some negative peaks of the signal will be clipped and the resulting distortion causes bit-error-rate (BER) performance degradation, even with a high bias [6]. ACO-OFDM converts the bipolar signal to its unipolar counterpart by clipping all negative values at zero and then modulating odd subcarriers only. However, a large part of bandwidth has been sacrificed to guarantee the asymmetrical property. Due to this, the spectral efficiency of DCO-OFDM is about twice as large as the spectral efficiency of ACO-OFDM for the same order of quadrature amplitude modulation (M-QAM). Instead of applying Hermitian symmetry and signal clipping, a simple idea has been presented in [7] to ensure real-valued OFDM signals by mapping the complex time domain (output of IFFT) into a real value, with the same symbols prior to QAM modulation in the frequency domain. The work in [8] presented a new coding scheme by employing zero cross correlation (ZCC) codes to achieve the same goal and to eliminate multiple access interference, but this required large set sizes and, hence, more bandwidth. Motivated by this and in order to fully utilize the available bandwidth, this paper proposes a new approach based on complex signal mapping (CSM) with the pairing function method to 


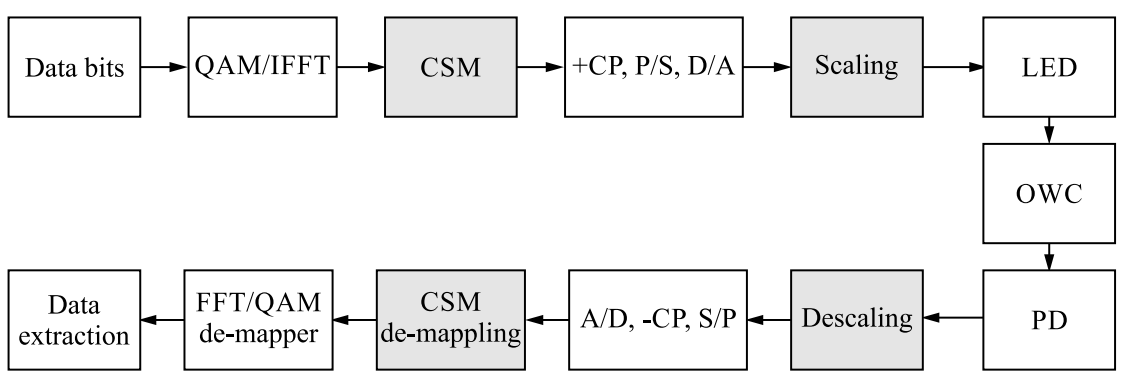

Fig. 1. System block of a Li-Fi transceiver based on OFDM with CSM.

encode a complex OFDM signal into a real-positive signal. This will result in improving the performance of $\mathrm{Li}-\mathrm{Fi}$ systems utilizing OFDM, in terms of bandwidth efficiency, BER performance and PAPR reduction.

This paper is organized as follows: in Section 2, system descriptions are explained. OFDM CSM procedures are given in Section 3. Detailed simulation results and a discussion are presented in Section 4, while our concluding remarks are provided in Section 5.

\section{System Description}

Figure 1 represents the proposed CSM-OFDM-based Li-Fi transceiver. The serial data bits are grouped into symbols and modulated using a QAM modulator. The modulated symbols are assigned to sub-carriers and then feed to an $\mathrm{N}$-points IFFT to provide the OFDM time domain signal. The simulation model is similar to the conventional optical OFDM-based VLC, only Hermitian symmetry is not needed for the CSM-OFDM system, and the real-valued time domain signal can be obtained using the CSM that maps the time domain complex OFDM symbols into real integer symbols. A fixed length cyclic prefix $(\mathrm{CP})$ is added to avoid any ISI, followed by parallel-to-serial (P/S) and digital-to-analog (D/A) conversions. The resulting signals are then scaled to be compatible with the LED dynamic range and pass through the optical wireless channel (OWC) with an additive white Gaussian noise (AWGN). On the receiver side, as shown in Fig. 1, data retrieval is the reverse of the process adopted on the transmitter side.

\section{Complex Signal Mapping Mechanism}

The general mapping operation for a complex signal may be expressed as [7]:

$$
x_{R}=f\left(x_{C}\right)=f(s, a, b),
$$

where $x_{C}$ is the complex signal output of IFFT, which may be written as $a+\mathrm{i} b$ in which $a$ is the real part and $b$ is the imaginary part, while $s$ represents all possible signs to each pair of real and imaginary samples, and it may be mapped according to Table 1.
Table 1

Sign mapping

\begin{tabular}{|c|c|c|}
\hline Possible sign pair & $\begin{array}{c}\text { Binary } \\
\text { representation }\end{array}$ & $\begin{array}{c}\text { Decimal } \\
\text { representation, } s\end{array}$ \\
\hline \hline-- & 00 & 0 \\
\hline-+ & 01 & 1 \\
\hline+- & 10 & 2 \\
\hline++ & 11 & 3 \\
\hline
\end{tabular}

Function $f$ maps the complex signal $x_{C}$ into real-valued $x_{R}$ with the help of the pairing function at the transmitter, and the estimated $\widetilde{x_{C}}$ can be extracted at receiver by de-mapping $\widetilde{x_{R}}$ using the inverse of the pairing function. However, the pairing function deals with non-negative integers [9]. Therefore, fractional $a$ and $b$ need to be modified and rounded to integers after taking their absolute values and multiplying them by a certain precision $P$ :

$$
A=\lfloor|a| \times p\rfloor, \quad\lfloor|b| \times P\rfloor,
$$

where $\lfloor$.$\rfloor is the round function which returns the closest$ integer value of the given non-integer.

Using the Cantor pairing function, the pair $[A, B]$ can be paired (encoded) into a single non-negative integer $C$ that is uniquely associated with that pair [9]. The pair function is given by:

$$
C=\text { pair }[A, B]=\frac{A^{2}+3 A+2 A B+B+B^{2}}{2} .
$$

The output of CSM is then to append the sign code with $C$, i.e. $x_{C S M}=[s: C]$. An example of the complete output codeword is shown in Table 2 for some selected IFFT outputs with $P$ of $10^{4}$.

The inverse function of unpair outputs the pair associated with each non-negative integer $C$ :

$$
A=C-\frac{w^{2}+w}{2} \text { and } B=\frac{w^{2}+3 w}{2}-C .
$$

With intermediate $w$ equaling:

$$
w=\left\lfloor\frac{-1+\sqrt{1+8 C}}{2}\right\rfloor \text {. }
$$


Table 2

CSM approach

\begin{tabular}{|c|c|c|c|c|c|}
\hline $\begin{array}{c}\text { Selected IFFT } \\
\text { output }\end{array}$ & $s$ & $\mathrm{~A}$ & $\mathrm{~B}$ & $\mathrm{C}$ & $\begin{array}{c}\text { Output codeword } \\
x_{C S M}=[s: C]\end{array}$ \\
\hline \hline$-0.0738-\mathrm{i} 0.1398$ & 0 & 738 & 1398 & 23145 & 023145 \\
\hline$-0.0245+\mathrm{i} 0.0682$ & 1 & 245 & 682 & 4346 & 14346 \\
\hline $0.0321-\mathrm{i} 0.0619$ & 2 & 321 & 619 & 4527 & 24527 \\
\hline $0.0340+\mathrm{i} 0.0849$ & 3 & 34 & 849 & 7225 & 37225 \\
\hline
\end{tabular}

If a free noise case is assumed, then the complex signal prior to FFT can be obtained by the reverse process that consists in unparing the received $C$, dividing $A$ and $B$ by $P$ to extract $a$ and $b$, and then combining them with their signs to form $a+\mathrm{i} b$ as mentioned earlier.

The resulting signal from mapping the complex $x_{C S M}$ signal needs to be scaled according to a specific scaling factor (SF), as shown later. Biasing is then performed by adding a DC-bias to the resulting signal to adjust the minimum value of the scaled signal to turn on voltage (TOV) of the LED. In this way, the maximum value of the signal will be fitted to the saturated point of the LED due to the deamplification process. The dynamic range of the resulting $D R x_{C S M}$ signal may be defined as:

$$
D R x_{C S M}=\max \left(x_{C S M}\right)-\min \left(x_{C S M}\right),
$$

where $\max \left(x_{C S M}\right)$ is the maximum value of $x_{C S M}$ and $\min \left(x_{C S M}\right)$ is its minimum value. LED dynamic range is:

$$
D R_{L E D}=I_{H}-I_{L}
$$

where $I_{H}$ is the maximum allowed LED drive current and $I_{L}$ corresponds to the minimum LED drive current according to the LED data sheet. The scaling factor SF can be obtained as:

$$
S F=\frac{D R_{L E D}}{D R x_{C S M}} .
$$

Hence, the de-amplified signal $x_{D}$ is:

$$
x_{D}=S F \times x_{C S M},
$$

The PAPR is defined as the ratio of the maximum transmitted signal power to the average power:

$$
P A P R=10 \log \frac{\max \left(x_{D}^{2}\right)}{E\left[x_{D}^{2}\right]},
$$

where $\max _{D}^{2}$ is the maximum value of the de-amplified signal power, $E[\cdot]$ is the average of those values [7]. The complementary cumulative distribution function (CCDF) of PAPR is the most commonly used measure for illustrating the PAPR reduction and it is used here to evaluate PAPR reduction.

The optimum biasing level may be obtained via:

$$
D C_{\text {bias }}=\mathrm{TOV}-\min \left(x_{D}\right) .
$$

Finally, the LED input signal can be obtained after shifting above signal to its TOV:

$$
x_{L E D}=x_{D}+D C_{b i a s}=x_{D}+\mathrm{TOV}-\min \left(x_{D}\right) .
$$

The spectral efficiency is defined as the amount of information bits transmitted per time unit per available bandwidth [10]. As the bandwidth is fully utilized, the SE of CSM-OFDM-based Li-Fi is twice that of DCO-OFDM, and four times that of ACO-OFDM. For N-FFT points and $M$ QAM order, the SE of Li-Fi system based on CSM-OFDM $\eta_{C S M}$ is:

$$
\eta_{C S M}=2 \eta_{D C O}=4 \eta_{A C O}=\frac{N-2}{N+N_{C P}} \log _{2} M
$$

where $\eta_{D C O}[\mathrm{bps} / \mathrm{Hz}]$ is the $S E$ of DCO-OFDM system, $\eta_{A C O}$ is the $S E$ of ACO-OFDM system and $N_{C P}$ is the CP length.

\section{Results and Discussion}

In this section, simulation results of the proposed scheme, related to spectral efficiency, PAPR and BER performance, are discussed and compared with conventional OFDM approaches in Li-Fi systems. The simulation is carried out using Matlab with 16 QAM and 128 subcarriers. CSM scheme processing is applied to the time domain signal after the IFFT, to obtain a real non-negative signal without employing the Hermitian symmetry to the input signal to fully utilize the available bandwidth in a Li-Fi network, as shown in Fig. 2. It may be noted that there is no distortion as all signals $\left(x_{C S M}, x_{D}\right.$ and $\left.x_{L E D}\right)$ have the same waveform. In fact, they are just scaled and shifted.

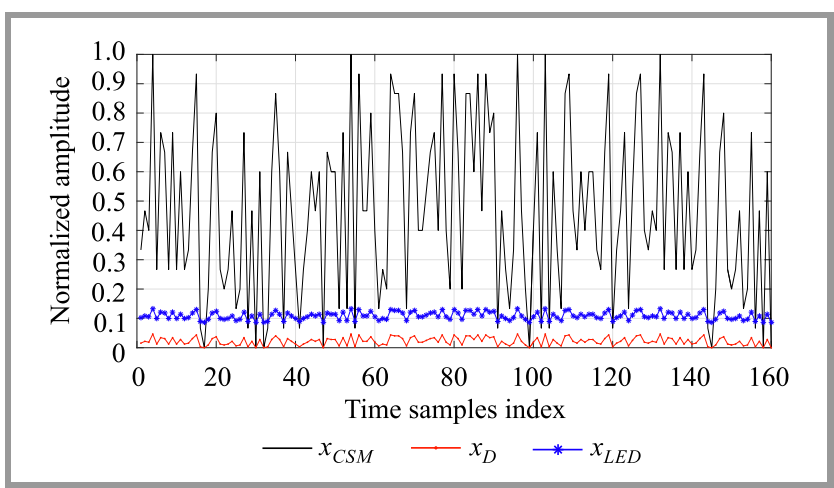

Fig. 2. Time domain signals of an OFDM-based Li-Fi system.

Spectral efficiency performance, as a function of modulation order, for different schemes using 128 FFT/IFFT subcarriers, is illustrated in Fig. 3. The proposed CSMOFDM avoids the use of Hermitian symmetry, therefore saving bandwidth compared to conventional DCO-OFDM and ACO-OFDM systems. It is clear that the proposed CSM-OFDM scheme outperforms other methods in terms of spectral efficiency for all QAM orders. This is because all available bandwidth has been fully utilized in the case of the proposed CSM-OFDM. DCO-OFDM imposes Hermitian symmetry to generate real-time domain signals and, 
hence, spectral efficiency is reduced by half in comparison to CSM-OFDM, while ACO-OFDM uses only one quarter of the available bandwidth. Hence, its spectral efficiency is also one quarter of the proposed CSM-OFDM-based Li-Fi system.

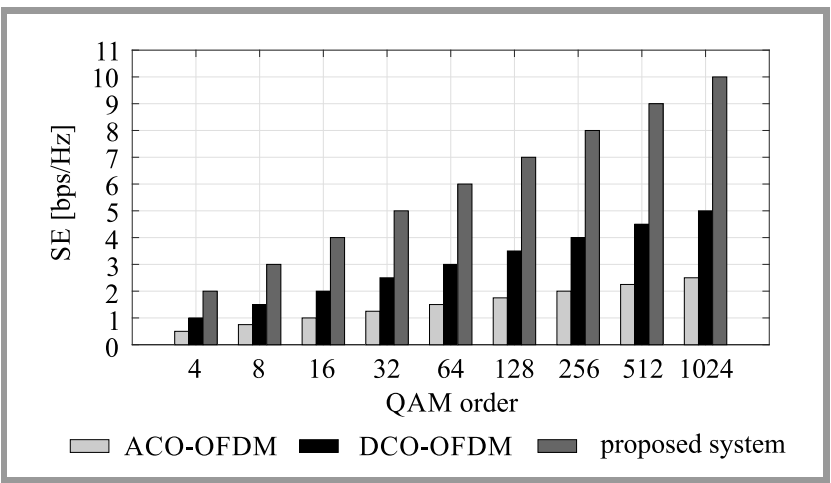

Fig. 3. Spectral efficiency versus modulation order for different schemes.

In order to make a fair comparison between the proposed CSM-OFDM scheme, ACO-OFDM and DCO-OFDM, simulations have been performed with the same size of IFFT and same M-QAM, i.e. IFFT size is 128 and 16-QAM is used for signal modulation for all optical OFDM formats. Figure 4 shows the CCDF comparison of PAPR for CSM-OFDM, ACO-OFDM and DCO-OFDM-based Li-Fi systems. CCDF curves show that DCO-OFDM has a higher PAPR compared to ACO-OFDM and CSM-OFDM systems. Clearly, the CSM-OFDM scheme achieves acceptable PAPR reduction, compared to other methods. However, the proposed scheme has a slightly lower PAPR ( $0.2 \mathrm{~dB}$ gain reduction) compared to ACO-OFDM at around CCDF of 0.1 .

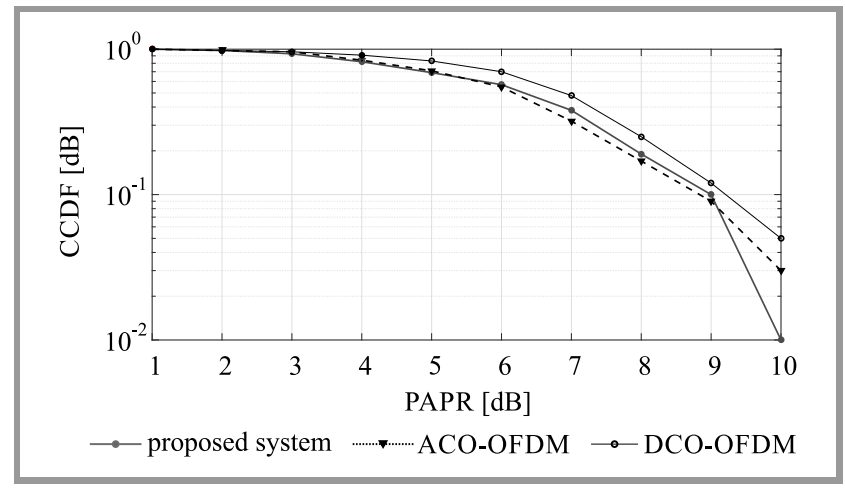

Fig. 4. CCDF performance of the OFDM-based Li-Fi signal with different approaches.

In particular, at $\mathrm{CCDF}=10^{-1}$, the PAPR threshold values of ACO-OFDM, CSM-OFDM and DCO-OFDM are 8.8, 9 and $9.2 \mathrm{~dB}$, respectively. Figure 5 shows that the proposed scheme also has a good BER performance. It is clear that DCO-OFDM outperforms other systems for all SNR values. The CSM-OFDM achieves better BER than ACO-OFDM for SNR of $19 \mathrm{~dB}$.

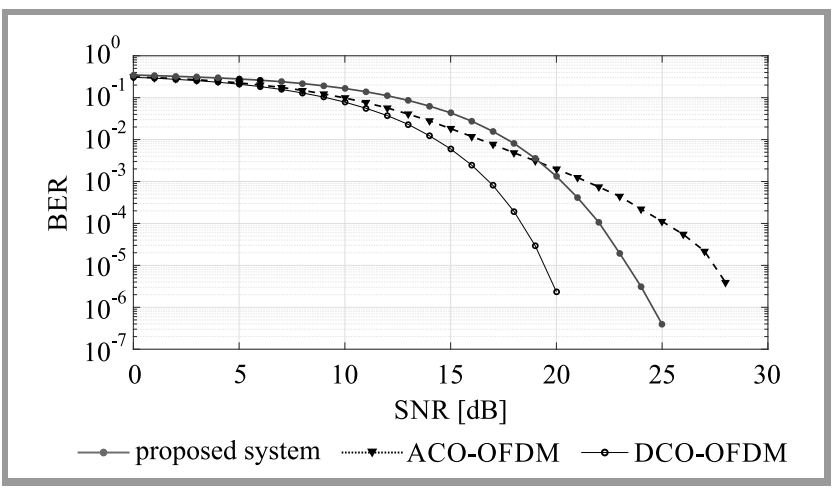

Fig. 5. Comparison of BER performance of OFDM-based Li-Fi signal with different approaches.

In fact, CSM-OFDM is more sensitive to channel change than other two systems at low SNR values, and it is suitable for high SNR when the channel is more slowly timevarying, which is the case in optical wireless communication systems.

\section{Conclusion}

In this paper, a new approach that avoids the use of Hermitian symmetry and signal clipping for transmitting OFDM signals is developed to increase the bandwidth efficiency of Li-Fi networks. The proposed CSM scheme encodes complex OFDM signals into real and positive values and decodes them prior to FFT at the receiver side, with the help of the pairing function mapping concept. The proposed CSM-OFDM-based Li-Fi system achieves double the spectral efficiency of DCO-OFDM and four times the spectral efficiency of ACO-OFDM systems. Simulation results show that the proposed CSM-OFDM scheme achieves performance that is comparable to ACO-OFDM and DCOOFDM, with a much lower bandwidth in terms of PAPR reduction and BER. In order to obtain further improvement in spectral efficiency of Li-Fi systems, the concepts of encoding the complex OFDM signal into real and nonnegative values for the IM/DD system need to be studied and will be included in future works.

\section{References}

[1] "Li-Fi market size forecast worth $\$ 75.5$ billion by 2023" [Online]. Available: https://www.gminsights.com/pressrelease/LiFi-market

[2] "IEEE 802.15 WPAN Task Group 13 (TG13) Multi-Gigabit/s Optical Wireless Communications" [Online]. Available: http://www.ieee802.org/15/pub/TG13.html

[3] "High speed indoor visible light communication transceiver - System architecture, physical layer and data link layer specification", [811-WP1] Draft new Recommendation ITU-T G.vlc [Online]. Available: https://www.itu.int/md/T13-SG15-160919-TD-WP1-0811/en

[4] J. Armstrong and A. Lowery, "Power efficient optical OFDM", Electron. Lett., vol. 42, no. 6, pp. 370-372, 2006 (doi: 10.1049/el:20063636).

[5] O. Gonzalez, R. Perez-Jimenez, S. Rodriguez, J. Rabadan, and A. Ayala, "Adaptive OFDM system for communications over the indoor wireless optical channel", IEE Proc. - Optoelec., vol. 153, pp. 139-144, 2006 (doi: 10.1049/ip-opt:20050081). 
[6] Y. Aimer, B. S. Bouazza, S. Bachir, and C. Duvanaud, "Interleaving technique implementation to reduce PAPR of OFDM signal in presence of nonlinear amplification with memory effects", J. of Telecommun. and Inform. Technol., no. 3, 2018 (doi: 10.26636/jtit.2018.123517).

[7] A. A. Abdulkafi, M. Y. Alias, and Y. S. Hussein, "A novel approach for PAPR reduction in OFDM-based visible light communications", in Proc. Int. Conf. on Plat. Technol. and Service PlatCon 2017, Busan, South Korea, 2017, pp. 1-4 (doi: 10.1109/PlatCon.2017.7883678).

[8] M. Addad and A. Djebbari, "A new code family for QS-CDMA visible light communication systems", J. of Telecommun. and Inform. Technol., no. 3, 2018 (doi: 10.26636/jtit.2018.124318).

[9] S. Wolfram, A New Kind of Science. Wolfram Media, 2002 (ISBN13: 9781579550080)

[10] D. Tsonev, M. S. Islim, and H. Haas, "OFDM-Based visible light communications", in Optical Wireless Communications. An Emerging Technology, M. Uysal, C. Capsoni, Z. Ghassemlooy, A. Boucouvalas, and E. Udvary, Eds. Springer, 2016, pp. 255-298 (doi: 10.1007/978-3-319-30201-0_12)

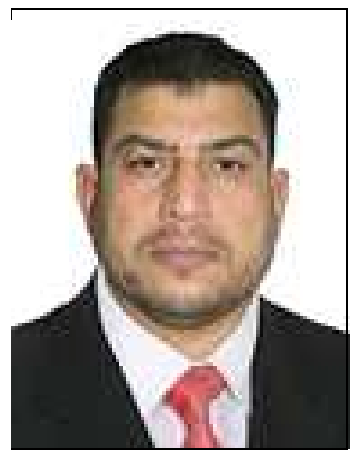

Ibrahim K. Sileh received his B.Sc. degree in Electrical Engineering (majoring in Electronic and Communications) from AlMosul University, Mosul, Iraq, in 1993, and M.Sc. degree in Communication Engineering from University of Technology: Al-Rasheed College, Baghdad, Iraq, in 2001. He received his Ph.D. in Communication Engineering from University of Southern Queensland, Australia in 2014. He is a staff member at the College of Engineering, Tikrit University, Iraq. His research interests are in wireless communications, including MIMO, Cooperative communications, heterogeneous networks, coding and OFDM.

(i) https://orcid.org/0000-0002-6484-0055

E-mail: ibrahim_aljebori@yahoo.com

Electrical Engineering

Tikrit University

Salahaddin, Iraq

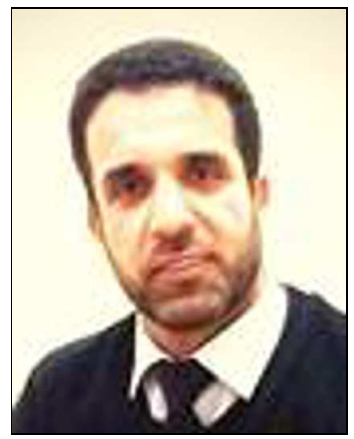

Ayad Atiyah Abdulkafi received his B.Sc. and M.Sc. degrees in Electrical Engineering (majoring in Telecommunications) from Al-Mustansisiriya University, Baghdad, Iraq, in 2001 and 2004, respectively. He then received his Ph.D. in Wireless Communication Engineering from University Tenaga National (UNITEN), Malaysia in 2015. He was a postdoctoral research Fellow at the Faculty of Engineering, Multi- media University, Cyberjaya, Malaysia. He is a staff member at the Faculty of Electrical Engineering, Tikrit University, Iraq. His research interests are in wireless communications, including, LTE, heterogeneous networks, energyefficient wireless network design, Green Cellular Networks, OFDM, optical wireless communications, and Visible Light Communication.

(iD) https://orcid.org/0000-0002-1160-6011

E-mail: al.ayad@yahoo.com

Electrical Engineering

Tikrit University

Salahaddin, Iraq

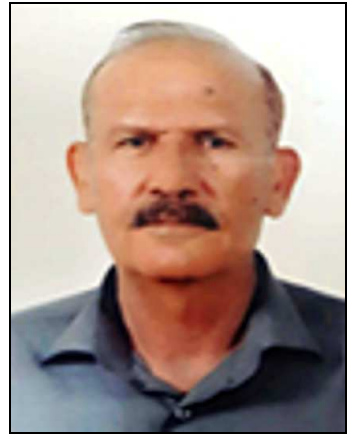

Mohamed Khalf Hussein received his B.Sc. degree in Electronics Engineering from AirMilitary Engineering Academy, Faculty of Electronics, Sarajevo, Yugoslavia, in 1982 and M.Sc. in Electronics Engineering, from the University of Belgrade, Electrical Engineering College, Belgrade, Yugoslavia, in 1984 . He is currently a staff member at the Faculty of Electrical Engineering, Shirqat, Tikrit University, Iraq. His research interests are in wireless communications, optical communications networks, coding, and OFDM.

E-mail: muh_kh48@yahoo.com

Electrical Engineering

Tikrit University

Salahaddin, Iraq

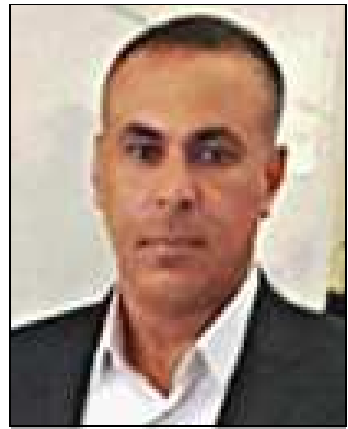

Saad Mshhain Hardan received his B.Sc. and M.Sc. degrees in Electrical and Electronic Engineering, Communication Engineering from the University of Technology, Baghdad, Iraq in 1997 and 2005, respectively. $\mathrm{He}$ is a staff member at the College of Engineering, Tikrit University, Iraq. He is currently a Ph.D. candidate at Altinbas University, Turkey. His research interests are in Radio Wireless Communication, Optical Wireless Communication, Visible Light Communication, Digital Communication, and Wireless Communication Networks.

(iD) https://orcid.org/0000-0003-2395-2525

E-mail: saadmhf@yahoo.com

Electrical Engineering

Tikrit University

Salahaddin, Iraq 